\title{
Overexpression of mimecan in human aortic smooth muscle cells inhibits cell proliferation and enhances apoptosis and migration
}

\author{
HUI-JIE ZHANG ${ }^{1 *}$, JING WANG $^{1 *}$, HUI-FANG LIU ${ }^{1}$, XIAO-NA ZHANG $^{2}$, MING ZHAN $^{2}$ and FENG-LING CHEN ${ }^{1}$ \\ ${ }^{1}$ Department of Endocrinology, Third People's Hospital Affiliated to Shanghai Jiao Tong University School of Medicine, \\ Shanghai 201900; ${ }^{2}$ Shanghai Institution of Endocrinology, Ruijin Hospital Affiliated to Shanghai Jiao Tong \\ University School of Medicine, Shanghai 200025, P.R. China
}

Received April 4, 2014; Accepted January 12, 2015

DOI: $10.3892 /$ etm.2015.2444

\begin{abstract}
The pathogenesis of atherosclerosis is multifactorial. The proliferation and migration of vascular smooth muscle cells (VSMCs) are significant in the genesis and development of atherosclerosis plaques, and the degradation of VSMCs plays a crucial role in the process. Mimecan is a member of the Keratan sulfate family of proteoglycans, which are leucine-rich proteoglycans. It has been demonstrated that mimecan is associated with arteriogenesis and atherosclerosis. In the present study, the effect of mimecan on the characteristics of cultured human aortic smooth muscle cells (HASMCs) was investigated. In vitro, human mimecan was stably overexpressed in HASMCs using a lentiviral system. It was observed that the proliferation rate of HASMCs transduced with mimecan was lower compared with that of control cells; overexpression of mimecan induced HASMC apoptosis. To determine the effect of mimecan on HASMC migration, a Transwell cell culture chamber and sterile cloning cylinder assays were used, and it was noted that mimecan enhanced the migration of HASMCs horizontally and vertically. These data indicated that mimecan may be involved in the pathogenesis of atherosclerosis by regulating the proliferation, apoptosis and migration of VSMCs.
\end{abstract}

\section{Introduction}

Atherosclerosis is a complex disease involving numerous cells, including macrophages, lymphocytes, neutrophils, endothelial cells and vascular smooth muscle cells (VSMCs) (1). During the last decades, a large number of studies have suggested

Correspondence to: Dr Feng-Ling Chen, Department of Endocrinology, Third People's Hospital Affiliated to Shanghai Jiao Tong University School of Medicine, 280 Mohe Road, Shanghai 201900, P.R. China

E-mail: phdflchen@163.com

\section{${ }^{*}$ Contributed equally}

Key words: mimecan, human aortic smooth muscle cells, proliferation, apoptosis, migration that VSMCs may play a key role in the genesis and development of atherosclerosis $(1,2)$. At an early stage, macrophages derived from peripheral blood mononuclear cells secrete various cytokines, some of which promote VSMC migration from the tunica media to the subendothelial region, where they proliferate and take up highly oxidized low-density lipoprotein, forming foam cells. Over time, the proliferation and migration of VSMCs result in the expansion of the lesion. Then, with the death of the foam cells and further accumulation of lipids, the advanced fibro-fatty lesion develops, which is mainly composed of accumulated VSMCs and smooth muscle cell (SMC)-derived extracellular matrix (ECM) (2). In addition, a number of cytokines, chemokines and growth factors, including monocyte chemotactic protein-1 (MCP-1) and angiotensin II (Ang II), are involved in the regulation of VSMCs in atherosclerosis $(3,4)$.

Mimecan, also known as osteoglycin or osteoinductive factor, was originally isolated from bovine bone and belongs to the family of small leucine-rich proteoglycans (SLRPs) $(5,6)$. The structural hallmarks of SLRPs are leucine-rich repeats (LRRs) and a cysteine-rich cluster in the N-terminal region, which have highly conserved spacing and are involved in the regulation of cell proliferation and differentiation $(7,8)$. Mimecan demonstrates a unique sequence of cysteine-rich regions and six LRRs. There is increasing evidence demonstrating that mimecan is involved in arteriogenesis and atherosclerosis. Shanahan et al (9) demonstrated that mimecan is a novel marker of differentiated VSMCs and may be an essential component of the normal vascular matrix. Using quantitative polymerase chain reaction (qPCR), mimecan was observed to be decreased in the media of vessel walls and increased in the activated endothelium and thickened neointima (9). Immunohistochemistry experiments revealed that mimecan accumulated in the leading edge of migrating SMCs in rabbit atherosclerotic lesions (10). These results indicated that mimecan played a role in atherosclerosis (10). A previous study reported that mimecan is distributed in the adventitia of collateral arteries, expressed mostly in SMCs and perivascular fibroblasts in the rabbit femoral artery ligation model. The observation also confirmed that mimecan is downregulated in arteriogenesis (11). Another study demonstrated that mimecan may play a significant role in the regulation of cellular growth and differentiation (12). It has been reported 
that mimecan gene expression is downregulated by certain growth factors and cytokines associated with vascular injury, and activated by the tumor suppressor protein p53 $(13,14)$.

Although the existing literature indicates that mimecan plays a role in arteriogenesis and cellular growth, there is no direct evidence of the effect of mimecan on cell proliferation, apoptosis and migration in VSMCs. The purpose of the present study was to observe the effect of mimecan on cultured human aortic smooth muscle cells (HASMCs) in vitro.

\section{Materials and methods}

HASMC cultures. HASMCs were grown in culture flasks in medium 231 with smooth muscle growth supplement (SMGS). Cells, medium 231 and SMGS were all purchased from Cascade Biologics (Portland, OR, USA). The cells were incubated at $37^{\circ} \mathrm{C}$ in a humidified $5 \% \mathrm{CO}_{2}$ atmosphere. HASMCs were subcultured when the cells reached approximately $70-80 \%$ confluence. Cells from passages 4 to 10 were used for experiments.

Stable transfection. Full-length human mimecan cDNA was generated by PCR amplification, incorporating XhoI and $B g l \mathrm{II}$ (Takara, Kyoto, Japan) restriction sites and adding three FLAG tags. The primers for the full-length human mimecan were 5'-ACTGAGATCTATGAAGACTCTGCAGTCTACACTTCT CCTGTTACTGCTTGTGCCTCTGATAAAGCCAGCACCA CCAACCCAGCAGGACTCACGC-3' and 5'-CTGGCT CGAGTTACTTGTCGTCATCGTCTTTGTAGTCGATGTCA TGGTCTTTGTAGTCTCCGTCATGGTCTTTGTAGTCAA AGTATGACCCTAT-3'. The PCR product was ligated into the pMSCV-puromycin-IRES-GFP (PIG; Clontech, Mountain View, CA, USA) vector, resulting in a PIG lentiviral construct. Recombinant lentiviruses were produced by transient co-transfection of three plasmids in HEK293T (ATCC, Manassas, VA, USA) cells using Lipofectamine 2000 (Invitrogen, Carlsbad, CA, USA) according to the manufacturer's instructions. The three plasmids were h-mimecan-3FLAG-PIG or empty vector $\mathrm{PIG}$, the envelope protein VSV-G and the packaging protein gag-pol (Clontech). Infectious lentivirus was harvested at $48 \mathrm{~h}$ post-transfection and filtered through $0.45 \mu \mathrm{m}$-pore cellulose acetate filters (Millipore, Billerica, MA, USA). HASMCs were plated on a six-well plate, and the medium was changed to perfect medium (medium 231 with SMGS) without antibiotics prior to transduction. When the cells reached $60 \%$ confluence, the lentiviruses and $8 \mu \mathrm{g} / \mathrm{ml}$ polybrene (Invitrogen) were added to each well. Following incubation for $6-8 \mathrm{~h}$ at $37^{\circ} \mathrm{C}$, the medium containing lentiviruses was removed and replaced with perfect medium. When the cells reached $90 \%$ confluence, $1 \mu \mathrm{g} / \mathrm{ml}$ puromycin (Gibco, New York, NY, USA) was added for 4 days. Human mimecan expression was verified by reverse transcription (RT)-qPCR and western blot analysis.

$R T$ - $q P C R$. Total RNA was extracted from transduced cells using TRIzol (Invitrogen). The cDNA was synthesized from $1 \mu \mathrm{g}$ RNA using a Takara reverse transcription kit according to the manufacturer's instructions. RT-qPCR was performed using SYBR-Green PCR Master mix (Takara) according to the manufacturer's instructions. The sense primer for GAPDH was 5'-GATCATCAGCAATGCCTCCTGC-3', and the anti- sense primer was 5'-GGTCATGAGTCCTTCCACGAT ACC-3'. The sense primer for mimecan was 5'-TACTTGGAC CATAATGCCCTGG-3', and the antisense primer was 5'-AAC TGGTGTCATTAGCCTTGC-3'. The resultant 127-bp fragment was confirmed by sequencing and comparing with the sequence of the human mimecan gene expression region in GeneBank.

Western blot analysis. When the cells reached $90 \%$ confluence, they were harvested to extract total cell protein. The proteins were separated by $10 \%$ SDS-PAGE and transferred to polyvinylidene difluoride membranes (Roche, Basel, Switzerland), after which the membranes were probed with the following antibodies: The primary antibody, anti-FLAG (1:1,000; Sigma-Aldrich, St. Louis, MO, USA); the horseradish peroxidase-coupled secondary antibody (1:2,000; DAKO, Glostrup, Denmark); and anti-GAPDH (1:10,000; Kangchen Raybiotech, Shanghai, China). The results were analyzed using the LAS-4000 system (Fujifilm, Tokyo, Japan).

Cell proliferation assay. Cell proliferation was measured by 3-(4,5-dimethylthiazol-2-yl)-2,5-diphenyltetrazolium bromide (MTT)assay.HASMCstransducedwithh-mimecan-3FLAG-PIG or empty vector were seeded at a density of $1 \times 10^{3}$ cells/well on 96-well plates. The cells were incubated at $37^{\circ} \mathrm{C}$ for $24 \mathrm{~h}$, following which $20 \mu \mathrm{l}$ methyl thiazolyl tetrazolium $(5 \mathrm{mg} / \mathrm{ml}$ MTT, Sangon Biotech, Shanghai, China) was added into each well and incubated for $3-4 \mathrm{~h}$ at $37^{\circ} \mathrm{C}$. The medium was then carefully removed, and $200 \mu \mathrm{l}$ dimethyl sulphoxide (DMSO, Invitrogen) was added to each well. Spectrophotometric readings were normalized using cell-free media as a blank. The plate was read spectrophotometrically by measuring the absorbance of the dye at a wavelength of $490 \mathrm{~nm}$. Four wells per treatment were used for the assay, and each experiment was performed in triplicate.

Cell apoptosis assay. To measure the effect of overexpression of mimecan on HASMC apoptosis, the cells were seeded in six-well plates at a density of $1 \times 10^{4}$ per well and incubated for $36 \mathrm{~h}$ at $37^{\circ} \mathrm{C}$. The cells were then harvested. Cells from each well were suspended in $195 \mu \mathrm{l}$ binding buffer, and $5 \mu \mathrm{l}$ Annexin V-phosphatidylethanolamine (PE) was added. The mixture was incubated for $10 \mathrm{~min}$ at room temperature in the dark and then harvested and suspended in $200 \mu \mathrm{l}$ binding buffer according to the manufacturer's instructions (Beyotime Institute of Biotechnology, Shanghai, China). The cells were subjected to flow cytometry to measure the apoptosis rate with a cytometer (Beckman Coulter, Brea, CA, USA). Experiments were repeated three times.

Cell migration assay. The migration activity of cells was assayed in Transwell cell culture chambers (BD Biosciences, Franklin Lakes, NJ, USA) and sterile cloning cylinders (Bel-Art Products, Wayne, NJ, USA). HASMCs $\left(2 \times 10^{3}\right)$ were resuspended in $200 \mu \mathrm{l}$ condition medium (medium 231 with $0.1 \%$ bovine serum albumin). The cell suspension was added to the upper compartment of the chamber, and incubated with $700 \mu \mathrm{l}$ perfect medium (medium 231 with SMGS) in the lower compartment for $24 \mathrm{~h}$ at $37^{\circ} \mathrm{C}$. Following incubation, the inserts were removed from the wells, and the cells on the 
A

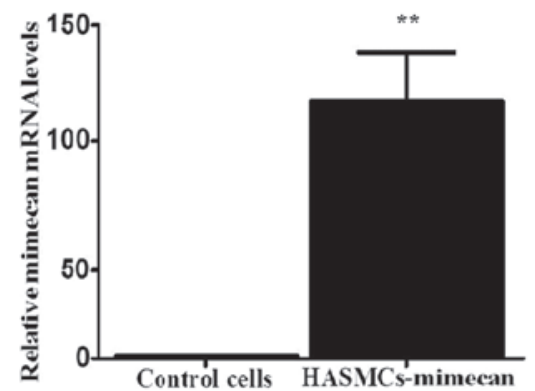

B

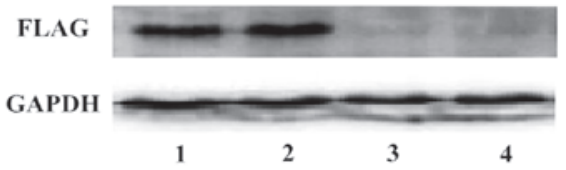

Figure 1. Level of mimecan mRNA and protein expressed following transduction with h-mimecan-3FLAG-puromycin-IRES-GFP (PIG) or empty vector. Human aortic smooth muscle cells (HASMCs) were transduced with h-mimecan-3FLAG-PIG or empty vector via a lentivirus. The increase in mimecan mRNA expression is presented as the fold change in the expression of mimecan normalized to GAPDH relative to the control measured by reverse transcrption-quantitative polymerase chain reaction analysis (A). FLAG protein expression in HASMCs transduced with h-mimecan-3FLAG-PIG plasmid is positive while that in control cells is negative (B). 1 and 2, HASMC-mimecan (HASMCs transduced with h-mimecan-3FLAG-PIG plasmid); 3 and 4, control cells (HASMCs transduced with empty vector). ${ }^{* * *} \mathrm{P}<0.01$ vs. control cells.

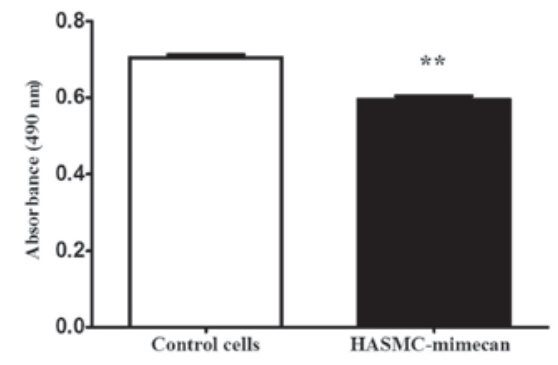

Figure 2. Effect of mimecan on human aortic smooth muscle cell (HASMC) proliferation. Each result was expressed as the absorbance at $490 \mathrm{~nm}$. Every sample was corrected by cell-free media. ${ }^{* *} \mathrm{P}<0.01$ vs. control cells.

upper surface of the filters were removed by cotton swabs. The filters were fixed with $4 \%$ paraformaldehyde (Sangon Biotech) and stained with crystal violet staining reagent (Beyotime Institute of Biotechnology). Migrated cells were manually counted using a light microscope. Cells in nine random high-power fields were counted for each migration well to determine the number of migrated cells. HASMCs $\left(1 \times 10^{3}\right)$ were resuspended in $50 \mu 1$ condition medium. The suspension was added to the inner wall of the sterile cloning cylinders, and the edge of the sterile cloning cylinders were marked using a syringe needle. After the cells adhered, the sterile cloning cylinders were removed and $500 \mu 1$ perfect medium was added to the 12 -well plate. The cells were incubated for $24 \mathrm{~h}$ at $37^{\circ} \mathrm{C}$ to evaluate their migration ability. The migrated distance $(\mathrm{mm})$ was measured at ten points along the edge of the sterile cloning cylinder using the Image Pro Plus, version 6.0 (Media Cybernetics, Inc., Bethesda, MA, USA) image analysis system. Each assay was performed in triplicate.
A
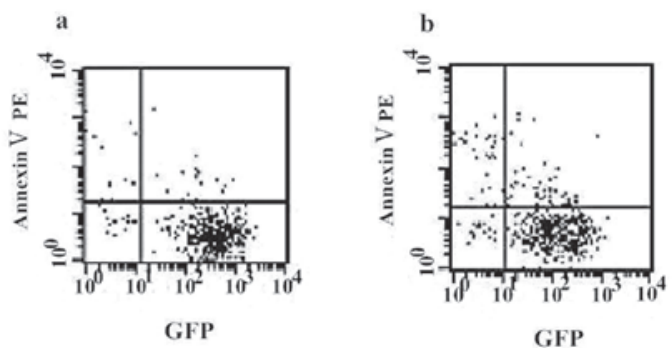

GFP

B

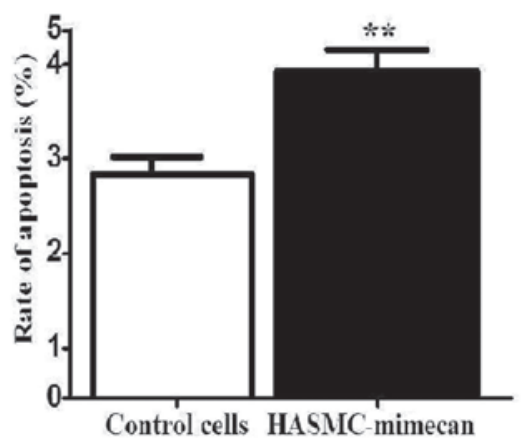

Figure 3. Effect of mimecan on human aortic smooth muscle cell (HASMC) apoptosis. (A) The effect of mimecan on HASMC apoptosis was measured by flow cytometry analysis of staining of phosphatidylethanolamine (PE)-labeled Annexin V. The cells in the upper right quadrant were positive for Annexin V and green fluorescent protein (a, control cells; b, HASMC-mimecan). (B) Apoptosis rate of HASMC-mimecan or control cells according to the results of the flow cytometry assay. ${ }^{*} \mathrm{P}<0.05$ vs. control cells.

Statistical analysis. Data are presented as the means \pm standard deviation. Statistical analyses were performed using SPSS 17.0 software (SPSS, Inc., Chicago, IL, USA). $\mathrm{P}<0.05$ and $\mathrm{P}<0.01$ were considered to indicate a statistically significant difference.

\section{Results}

Transduction of h-mimecan-3FLAG-PIG results in target-specific overexpression. To determine the effect of mimecan on HASMCs, a lentiviral system was used to stably overexpress mimecan in vitro. The HASMCs transduced with h-mimecan-3FLAG-PIG (HASMC-mimecan) demonstrated a 100 -fold increase in mimecan mRNA levels compared with control cells as determined by RT-qPCR (Fig. 1A; P<0.01). HASMCs transduced with empty vectors were used as control cells. The transduction of HASMCs with mimecan resulted in specific overexpression of mimecan protein, as determined by western blot analysis (Fig. 1B). The transduction efficiency was demonstrated by the expression of GFP (data not shown).

Mimecan inhibits proliferation of HASMCs. The effect of mimecan overexpression on the proliferation of HASMCs was measured by MTT assay. The proliferation rate was quantified by measuring the absorbance at $490 \mathrm{~nm}$. The results demonstrated that overexpression of mimecan decreased the proliferation rate by $16 \%(\mathrm{P}<0.01$; Fig. 2$)$.

Mimecan induces apoptosis in HASMCs. Cells undergoing apoptosis revealed translocation of phosphatidylserine (PS) to the outer leaflets of the plasma membrane. Apoptosis levels were measured by staining of Annexin V-PE targeted to PS. 
A

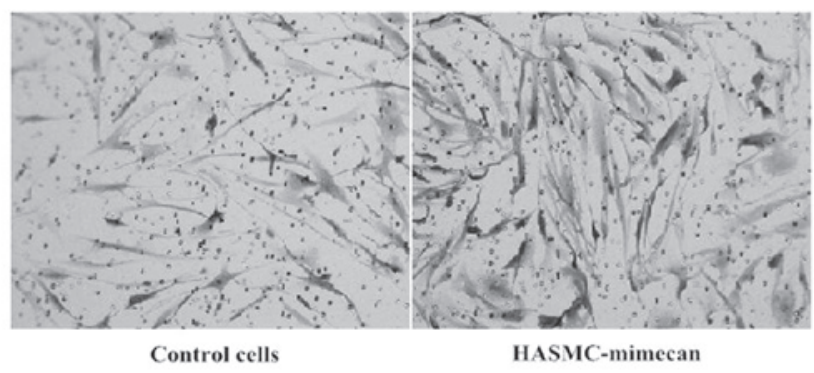

B

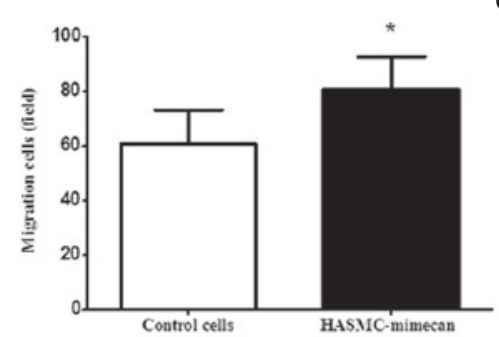

C

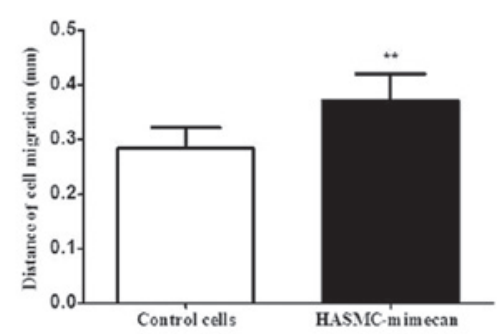

Figure 4. Effect of mimecan on human aortic smooth muscle cell (HASMC) migration. (A and B) HASMC migration was measured by Transwell cell culture chamber migration assay and sterile cloning cylinder assay. Overexpression of mimecan increased the basal migration of HASMCs 1.34-fold (82 cells/field) compared with that of control cells (61 cells/field). (C) In the sterile cloning cylinder migration assay, the HASMC-mimecan migration distance was greater $(0.37 \mathrm{~mm})$ compared with that of control cells $(0.28 \mathrm{~mm}) .{ }^{*} \mathrm{P}<0.05,{ }^{* *} \mathrm{P}<0.01 \mathrm{vs}$. the control cells.

The rate of apoptosis was assessed by flow cytometry. The results reveal that overexpression of mimecan increased the cell apoptosis rate compared with that of control cells (Fig. 3; $\mathrm{P}<0.05)$.

Mimecan enhances HASMC migration. The effect of mimecan on the migration of HASMCs was evaluated by the Transwell migration assay and sterile cloning cylinders migration assay, respectively. The Transwell migration assay demonstrated that mimecan enhances HASMCs migration. Overexpression of mimecan increased the basal migration of HASMCs 1.34-fold ( 82 cells/field) compared with that of control cells (61 cells/field) (Fig. 4A and B). The sterile cloning cylinder migration assay demonstrated the migration distance of HASMC-mimecan or control cells horizontally. As shown in Fig. 4C, the migration distance of HASMC-mimecan $(0.37 \mathrm{~mm})$ was greater than that of control cells $(0.28 \mathrm{~mm})$.

\section{Discussion}

The present study first established a cell model stably overexpressing mimecan in HASMCs using a lentivirus system. It provided a new cell model to investigate the function of mimecan in VSMCs. The HASMCs used in the present study were purchased from Cascade Biologics and have been used in a number of previous studies to investigate VSMCs $(15,16)$.

The SLRP gene family has been shown to be involved in the regulation of collagen fibrillogenesis, ECM assembly, cell growth, migration and adhesion $(8,17)$. It has been proven that mimecan, similar to other SLRP family members, has a role in regulating collagen fibrillogenesis, which is a component of ECM (18). Moreover, the biological scaffolds of ECM are essential for cell proliferation, adhesion and migration. Shanahan et al (9) and Scholz et al (19) reported that mimecan mRNA expression increases after VSMC prolifera- tion ceases. This result suggests that mimecan is associated with VSMC proliferation. It is also reported that knockdown of mimecan enhances primary cultured VSMC proliferation induced by FBS or Ang II (20). In addition, a large number of cytokines, including fibroblast growth factor-2, transforming growth factor- $\beta$, platelet-derived growth factor, Ang II and oncostatin $\mathrm{M}$, inhibit mimecan expression, and MCP-1 enhances the effect of these cytokines on mimecan $(9,11)$. Consistent with the previous observations, the present study clearly indicates that proliferation was significantly inhibited in the HASMCs that overexpressed mimecan. The functions and phenotypes of SMCs in naturally occurring lesions presented spatial-temporal patterns and dynamic aspects (21). VSMCs from atherosclerosis lesions at an early stage and vessel walls following balloon injury reveal high proliferation rates, while VSMCs from plaques at a late stage reveal low proliferation rates (22). Therefore, we concluded that if mimecan is involved in the formation of atherosclerosis through effects on HASMC proliferation, it mainly plays a role in the early stage.

Although numerous studies have observed that mimecan is associated with VSMC proliferation (9-11), it was not known whether mimecan was directly related to the apoptosis of VSMCs. In the present study, we investigated the effect of mimecan on HASMC apoptosis using Annexin V-PE staining of PS and observed that the apoptosis rate of HASMCs overexpressing mimecan was elevated compared with control cells. The apoptosis of VSMCs plays a subtle role in the development of atherosclerosis by decreasing the thickness of the neointima and fibrous cap (23). The fibrous cap consists of VSMCs and ECM. The proportion of apoptotic VSMCs is increased in the unstable plaque compared with the stable plaque. Loss of VSMCs by apoptosis leads to a thin fibrous cap of plaque by decreasing collagen biosynthesis, which increases the risk of plaque rupture (24). Therefore, it seems reasonable to speculate that increased mimecan expression may accelerate the 
formation of unstable atherosclerotic plaques by promoting VSMC apoptosis. However, the apoptosis of VSMCs can interrupt neointimal formation at defined time points and reverse the artery restenosis (25). Additionally, it has been confirmed that the consequences of VSMC apoptosis depend on the location of VSMCs in plaques and the stages of the atherosclerotic plaques. The complexity of VSMC apoptosis effects on atherosclerotic plaques may have led to the different results in the previous studies.

Migration of medial VSMCs into the intima is one of the key points in the development of atherosclerosis and vascular injury. A previous study indicated that mimecan plays a role in SMC migration. A different study demonstrated that mimecan protein accumulates at the front edge of migrating SMCs in atherosclerosis lesions (10). In the present study, Transwell cell culture chamber and sterile cloning cylinder migration assays were used to further confirm that mimecan promotes VSMC migration. The migration assays demonstrated that the overexpression of mimecan enhanced HASMC migration ability.

Matrix metalloproteinases (MMPs), which are produced by VSMCs and macrophages, play a role in vascular remodeling (26). MMPs, in particular MMP-2 and MMP-9, contribute to the pathogenesis of atherosclerosis by facilitating VSMC migration via matrix disruption (27). Numerous studies have demonstrated that MMP-2 and MMP-9 participate in the development of atherosclerosis by regulating the proliferation and migration of VSMCs (28-32). A knockout study demonstrated that MMP-9 is required for neointimal and arterial lesion formation by regulating the migration and proliferation of VSMCs $(26,28)$. Decorin, a member of the SLRP family, affects the production of MMPs. It has been demonstrated that overexpression of decorin increases protein levels of MMP-2 (33). The structure of mimecan is similar to decorin, as both of them are members of the SLRP family. The most salient feature is that decorin has ten LRRs, and mimecan has 6 LRRs. Therefore, we hypothesize that mimecan may regulate cell migration by affecting MMPs in a similar to decorin. Further study is required to investigate whether MMPs mediate the effect of mimecan on VSMC migration.

SMCs in the human aorta have long been known to be able to express unique contractile proteins, ion channels and signaling molecules, which are necessary for the cells' contractile function, and the SMCs have a low rate of proliferation and synthetic activity (34). In response to changes in the local microenvironment, SMCs undergo more subtle changes in phenotype and function, including proliferation, secretion, migration and differentiation (35). Changes in the local microenvironment affect vascular development, atherosclerosis formation and vascular rupture. During vascular development, VSMCs exhibit a high rate of proliferation, migration and production of ECM components, including collagen, elastin and proteoglycans that make up a major portion of the vessel wall, acquiring high contractile capabilities (17). These changes are known as 'phenotypic modulation' (34). In special conditions, including arterial wall injury or atherosclerosis, VSMCs, which have a low mitogenic activity level in the tunica media region of the blood vessel wall, may undergo progression from a contractile to a synthetic phenotype and begin proliferating in response to various growth factors or cytokines $(36,37)$.
The phenotypic state of SMCs is likely to be extremely complex as the functional role of SMCs changes during arteriogenesis or atherosclerosis. Animal experiments have also demonstrated that the expression and location of mimecan are different in VSMCs with different phenotypes during arteriogenesis (9-11). Taken together, we speculate that mimecan may play various roles in different stages of atherosclerosis through its involvement in the phenotypic modulation of VSMCs. However, we did not investigate the effect of mimecan on VSMCs in vivo, and further studies will be required to characterize molecular mechanisms of mimecan in VSMC function and atherosclerosis.

\section{Acknowledgements}

This study was funded by grants to FLC from the National Science Foundation of China (no. 30870954 and 81270908) and the Foundation for Science and Technology from the Medical College of Shanghai Jiao Tong University (no. YZ1054).

\section{References}

1. Ross R: The pathogenesis of atherosclerosis: a perspective for the 1990s. Nature 362: 801-809, 1993

2. Lusis AJ: Atherosclerosis. Nature 407: 233-241, 2000.

3. Rakesh K and Agrawal DK: Cytokines and growth factors involved in apoptosis and proliferation of vascular smooth muscle cells. Int Immunopharmacol 5: 1487-1506, 2005.

4. Selzman CH,Miller SA,Zimmerman MA,Gamboni-Robertson F, Harken AH and Banerjee A: Monocyte chemotactic protein-1 directly induces human vascular smooth muscle proliferation. Am J Physiol Heart Circ Physiol 283: H1455-H1461, 2002.

5. Bentz H, Chang RJ, Thompson AY, Glaser CB and Rosen DM: Amino acid sequence of bovine osteoinductive factor. J Biol Chem 265: 5024-5029, 1990.

6. Bentz H, Nathan RM, Rosen DM, Armstrong RM, Thompson AY, Segarini PR, Mathews MC, Dasch JR, Piez KA and Seyedin SM: Purification and characterization of a unique osteoinductive factor from bovine bone. J Biol Chem 264: 20805-20810, 1989.

7. Iozzo RV: The family of the small leucine-rich proteoglycans: key regulators of matrix assembly and cellular growth. Crit Rev Biochem Mol Biol 32: 141-174, 1997.

8. Iozzo RV: The biology of the small leucine-rich proteoglycans. Functional network of interactive proteins. J Biol Chem 274: 18843-18846, 1999.

9. Shanahan CM, Cary NR, Osbourn JK and Weissberg PL: Identification of osteoglycin as a component of the vascular matrix. Differential expression by vascular smooth muscle cells during neointima formation and in atherosclerotic plaques. Arterioscler Thromb Vasc Biol 17: 2437-2447, 1997.

10. Fernandez B, Kampmann A, Pipp F, Zimmermann R and Schaper W: Osteoglycin expression and localization in rabbit tissues and atherosclerotic plaques. Mol Cell Biochem 246: 3-11, 2003.

11. Kampmann A, Fernandez B, Deindl E, Kubin T, Pipp F, Eitenmuller I, Hoefer IE, Schaper W and Zimmermann R: The proteoglycan osteoglycin/mimecan is correlated with arteriogenesis. Mol Cell Biochem 322: 15-23, 2009.

12. Tasheva ES, Maki CG, Conrad AH, et al: Transcription activation of bovine mimecan by p53 through an intronic DNA binding site. Biochim Biophys Acta 1517: 333-338, 2001.

13. Long CJ, Roth MR, Tasheva ES, Funderburgh M, Smit R, Conrad GW and Funderburgh JL: Fibroblast growth factor-2 promotes keratan sulfate proteoglycan expression by keratocytes in vitro. J Biol Chem 275: 13918-13923, 2000.

14. Tasheva ES: Analysis of the promoter region of human mimecan gene. Biochim Biophys Acta 1575: 123-129, 2002.

15. Park SH, Koo HJ, Sung YY and Kim HK: The protective effect of Prunella vulgaris ethanol extract against vascular inflammation in TNF- $\alpha$-stimulated human aortic smooth muscle cells. BMB Rep 46: 352-357, 2013. 
16. Yisireyili M, Saito S, Niwa T, et al: Indoxyl sulfate-induced activation of (pro)renin receptor promotes cell proliferation and tissue factor expression in vascular smooth muscle cells. PLoS One 9: e109268, 2014

17. Svensson L, Oldberg A and Heinegård D: Collagen binding proteins. Osteoarthritis Cartilage 9 (Suppl A): S23-S28, 2001.

18. Tasheva ES, Koester A, Paulsen AQ, Garrett AS, Boyle DL, Davidson HJ, Song M, Fox N and Conrad GW: Mimecan/ osteoglycin-deficient mice have collagen fibril abnormalities. Mol Vis 8: 407-415, 2002.

19. Scholz D, Ito W, Fleming I, Deindl E, Sauer A, Wiesnet M, Busse R, Schaper J and Schaper W: Ultrastructure and molecular histology of rabbit hind-limb collateral artery growth (arteri0genesis). Virchows Arch 436: 257-270, 2000.

20. Gu XS, Lei JP, Shi JB, Lian WL, Yang X, Zheng X and Qin YW: Mimecan is involved in aortic hypertrophy induced by sinoaortic denervation in rats. Mol Cell Biochem 352: 309-316, 2011.

21. Owens GK, Kumar MS and Wamhoff BR: Molecular regulation of vascular smooth muscle cell differentiation in development and disease. Physiol Rev 84: 767-801, 2004.

22. Gordon D, Reidy MA, Benditt EP and Schwartz SM: Cell proliferation in human coronary arteries. Proc Natl Acad Sci USA 87: 4600-4604, 1990.

23. Bochaton-Piallat ML, Gabbiani F, Redard M, Desmouliere A and Gabbiani G: Apoptosis participates in cellularity regulation during rat aortic intimal thickening. Am J Pathol 146: 1059-1064, 1995.

24. Bauriedel G, Hutter R, Welsch U, Bach R, Sievert H and Luderitz B: Role of smooth muscle cell death in advanced coronary primary lesions: implications for plaque instability. Cardiovasc Res 41: 480-488, 1999.

25. Durand E, Mallat Z, Addad F, Vilde F, Desnos M, Guerot C, Tedgui A and Lafont A: Time courses of apoptosis and cell proliferation and their relationship to arterial remodeling and restenosis after angioplasty in an atherosclerotic rabbit model. J Am Coll Cardiol 39: 1680-1685, 2002.

26. Galis ZS and Khatri JJ: Matrix metalloproteinases in vascular remodeling and atherogenesis: the good, the bad, and the ugly. Circ Res 90: 251-262, 2002.
27. Bendeck MP, Zempo N, Clowes AW, Galardy RE and Reidy MA: Smooth muscle cell migration and matrix metalloproteinase expression after arterial injury in the rat. Circ Res 75: 539-545, 1994.

28. Abedi H and Zachary I: Signalling mechanisms in the regulation of vascular cell migration. Cardiovasc Res 30: 544-556, 1995.

29. Cho A and Reidy MA: Matrix metalloproteinase-9 is necessary for the regulation of smooth muscle cell replication and migration after arterial injury. Circ Res 91: 845-851, 2002.

30. Galis ZS, Johnson C, Godin D, Magid R, Shipley JM, Senior RM and Ivan E: Targeted disruption of the matrix metalloproteinase-9 gene impairs smooth muscle cell migration and geometrical arterial remodeling. Circ Res 91: 852-859, 2002.

31. Johnson JL, Dwivedi A, Somerville M, George SJ and Newby AC: Matrix metalloproteinase (MMP)-3 activates MMP-9 mediated vascular smooth muscle cell migration and neointima formation in mice. Arterioscler Thromb Vasc Biol 31: e35-e44, 2011.

32. Zempo N, Koyama N, Kenagy RD, Lea HJ and Clowes AW: Regulation of vascular smooth muscle cell migration and proliferation in vitro and in injured rat arteries by a synthetic matrix metalloproteinase inhibitor. Arterioscler Thromb Vasc Biol 16: 28-33, 1996.

33. Haj AL, Zen A, Lafont A, Durand E, Brasselet C, Lemarchand P, Godeau G and Gogly B: Effect of adenovirus-mediated overexpression of decorin on metalloproteinases, tissue inhibitors of metalloproteinases and cytokines secretion by human gingival fibroblasts. Matrix Biol 22: 251-258, 2003.

34. Owens GK: Regulation of differentiation of vascular smooth muscle cells. Physiol Rev 75: 487-517, 1995

35. Somlyo AP and Somlyo AV: Ca2+ sensitivity of smooth muscle and nonmuscle myosin II: modulated by $\mathrm{G}$ proteins, kinases, and myosin phosphatase. Physiol Rev 83: 1325-1358, 2003.

36. Chamley-Campbell J, Campbell GR and Ross R: The smooth muscle cell in culture. Physiol Rev 59: 1-61, 1979.

37. Schwartz CJ, Valente AJ, Sprague EA, Kelley JL, Cayatte AJ and Mowery J: Atherosclerosis. Potential targets for stabilization and regression. Circulation 86: III117-III123, 1992. 